\title{
An Improved Quad Flat Package for High Frequency SiP Applications
}

\author{
Haiyan $\operatorname{Sun}^{1,2}$ and Ling $\operatorname{Sun}^{2, *}$ \\ ${ }^{1}$ National ASIC System Engineering Research Center, \\ Southeast University, Nanjing 210096, People's Republic of China, \\ ${ }^{2}$ Jiangsu Provincial Key Lab of ASIC Design, \\ Nantong University, Nantong 226019, People's Republic of China \\ 1sun.yan@seu.edu.cn, ${ }^{2}$ sun.l@ntu.edu.cn
}

\begin{abstract}
This paper describes four cascade coplanar transmission line structures built into the quad flat pack (QFP) lead frame for high-frequency system in package (SiP) applications. Adjacent ground leads are used to provide return paths in a coplanar configuration, thereby minimizing impedance mismatch. Due to the complexity level in QFP structure, full-wave electromagnetic simulations have been performed to extract the S-parameter. Time Domain Reflectometry (TDR) is also used to help in understanding the contributing to the SiP structure. The SiP has also been fabricated and good correlation with electromagnetic simulations is also achieved.
\end{abstract}

Keywords: Quad flat package, system in package, electromagnetic modelling, coplanar transmission line structure

\section{Introduction}

The demand for domestic appliances as well as portable electronics has led the microelectronic industry to evolve into two complementary directions: system on chip (SoC) and SiP. Compared with SoC, SiP could enable the integration of heterogeneous functions (e.g., high-speed processors, RF devices, sensors, etc.) in a single compact package module. Also due to the demand of low-cost, QFP lead frame package is adopted to provide a doable solution to achieve SiP modules. The major problem when applying the low-cost QFP package to the high-frequency SiP applications is that the QFP package has much more impedance discontinuities than the high-end packages such as ball grid array (BGA). Parasitics of bonding wires and leads can cause a seriously degrade in SiP performance at higher frequency [1-4].

Figure 1 gives a selected traditional QFP80 lead frame for SiP application. Two dies are bonded to the paddle and encapsulated in plastic. Bond wires are used to provide connectivity from the dies to the leads. In order to illustrate the parasitic effects of the QFP80 package, Ansys HFSS is used to simulate the Z parameter of signal path S1. Figure 2 shows the simulated result when the signal $S 1$ is shorted to ground through a bond wire. Z11 rises dramatically due to the internal inductive reactances and exhibits a very strong resonance at $3.8 \mathrm{GHz}$, which makes the "short" appear as an open-circuit. Despite the concerns of high frequency loss, we believe that the signal transmission bandwidth of traditional QFP80 can be increased for high frequency SiP applications by further improved design. 


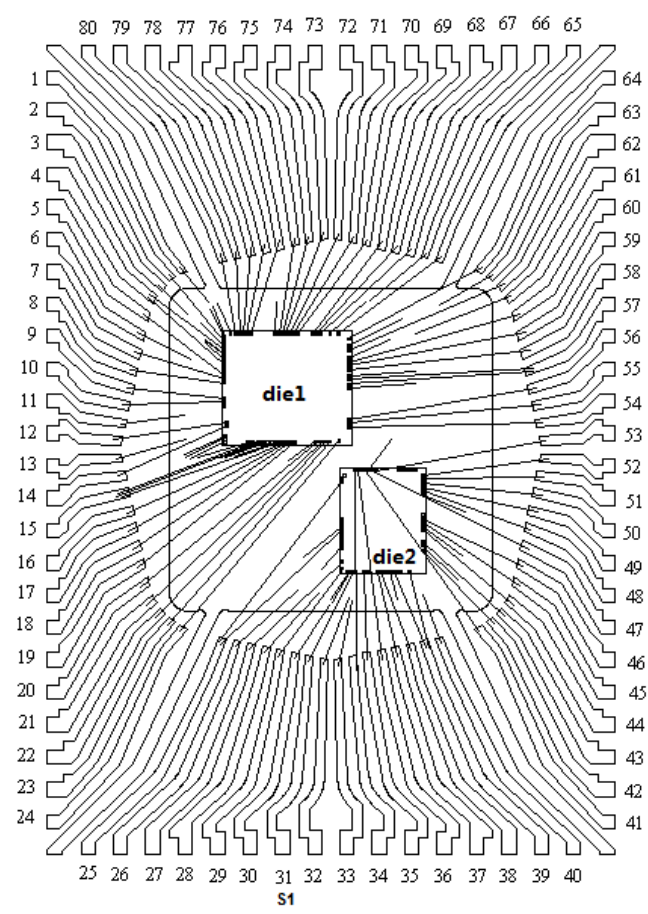

Figure 1. SiP Package based on a Traditional QFP80 Package

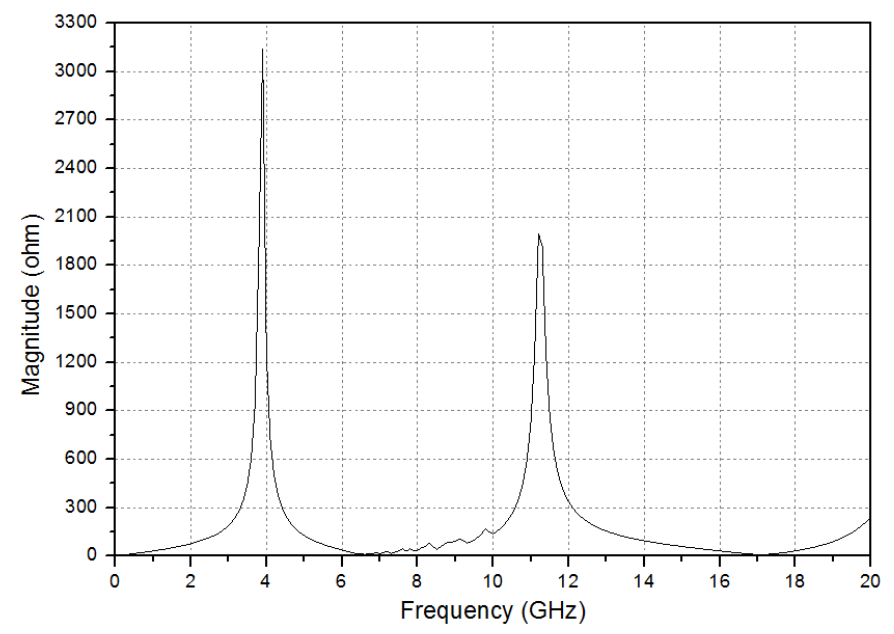

Figure 2. Simulated Result of Z11

In this paper, an improved QFP80 lead frame with four cascade coplanar transmission line structures have been developed. The SiP based on the improved QFP80 lead frame has also been fabricated and good correlation with electromagnetic simulations is achieved.

\section{Feasibility Analysis of the Coplanar Transmission Line Structure}

Usually, in low-frequency applications, the traditional QFP80 can be modelled as a lumped equivalent circuit. While in high-frequency SiP applications, the QFP80 lead frame defined by characteristic impedance and electrical length may provide a better transmission performance than lumped elements mutually coupled to neighboring traces. Figure 3 gives the two cross-sectional views and their corresponding equivalent circuit 
models respectively. In Figure 3 (a), $\mathrm{L}_{\text {bondwire }}$ and $\mathrm{R}_{\text {bondwire }}$ are the lumped inductance and resistance of the bond wire. $\mathrm{L}_{\text {lead }}, \mathrm{R}_{\text {lead }}$ and $\mathrm{C}_{\text {lead }}$ are the lumped inductance, resistance and capacitor of the lead. The terms $K_{\text {bondwire }}$ and $K_{\text {lead }}$ are the magnetic coupling coefficients between the bond wires and the leads, respectively. There is also a capacitive coupling term $\mathrm{C}_{\text {couple }}$ between leads. While in Figure 3 (b), the lumped elements of lead are substituted by a coplanar transmission line structure, and the raw coupling capacitance $\mathrm{C}_{\text {couple }}$ has been eliminated by tight coupling of the electric fields to the transmission line medium. If designed accurately, the PCB (printed circuit board) transmission line can be effectively extended to the bond wire location. So, the improved design of QFP80 is to make the discontinuity impedance of lead frame to be close to the characteristic impedance of the PCB transmission line.

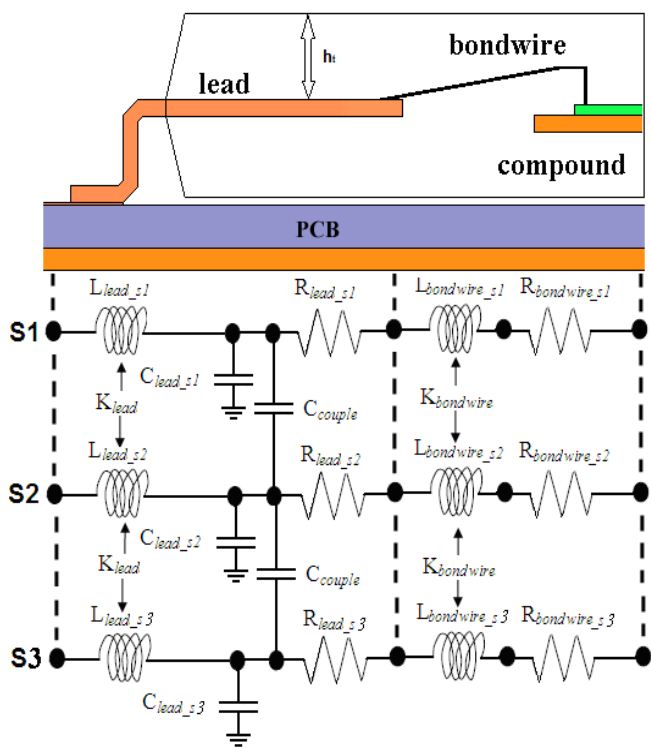

(a)

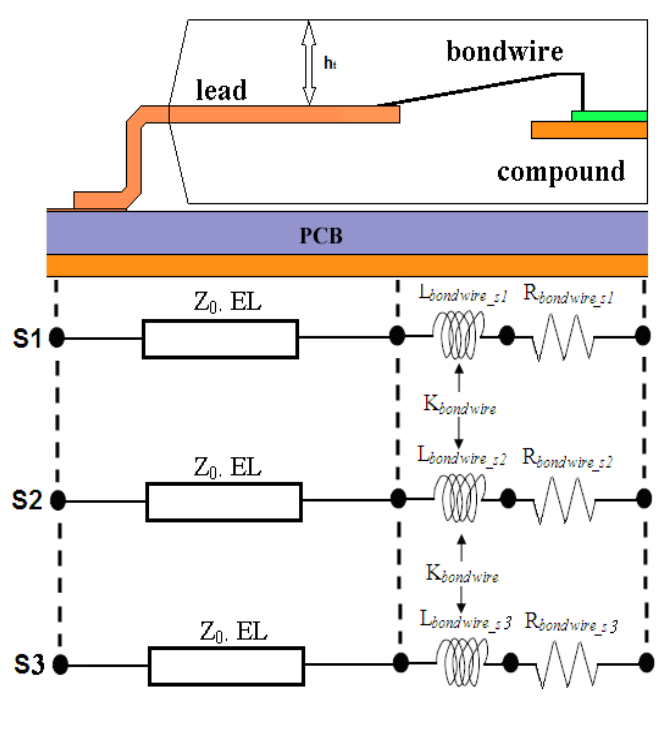

(b)

\section{Figure 3. (a) Simplified RLCM Equivalent Circuit of Lead Frame and (b) Transmission Line Equivalent Circuit of Lead Frame}

Figure 4 gives the two cross-sectional views of the traditional structure and proposed coplanar transmission line structure with finite irregular ground, respectively. As clearly seen in Figure 4 (b), with both adjacent conductors S1 and S3 grounded, the three leads S1, S2 and S3 have been used to construct a coplanar transmission line structure, similar to that of a coplanar waveguide. Compared with Figure 4 (a), two physical modifications transform the traditional QFP80 lead frame with coplanar transmission line structure. The first is that the grounded leads S1 and S3 have been extended to connect the paddle and the signal lead S2 has been extended to close the paddle. This change not only can provide a better ground shield to control crosstalk, but also can short the bond wire to achieve a lower inductance. The second is to set the width of signal lead and the space between leads to constant value. This change can be used to provide impedance control for signal trace. It should be noticed that the outside part of the signal lead is embedded in air $\left(\varepsilon_{\mathrm{r}} \approx 1\right)$, while the inside part is embedded in compound $\left(\varepsilon_{\mathrm{r}}=3.3\right)$. Therefore, the full lead should be designed as a cascade coplanar structure. 


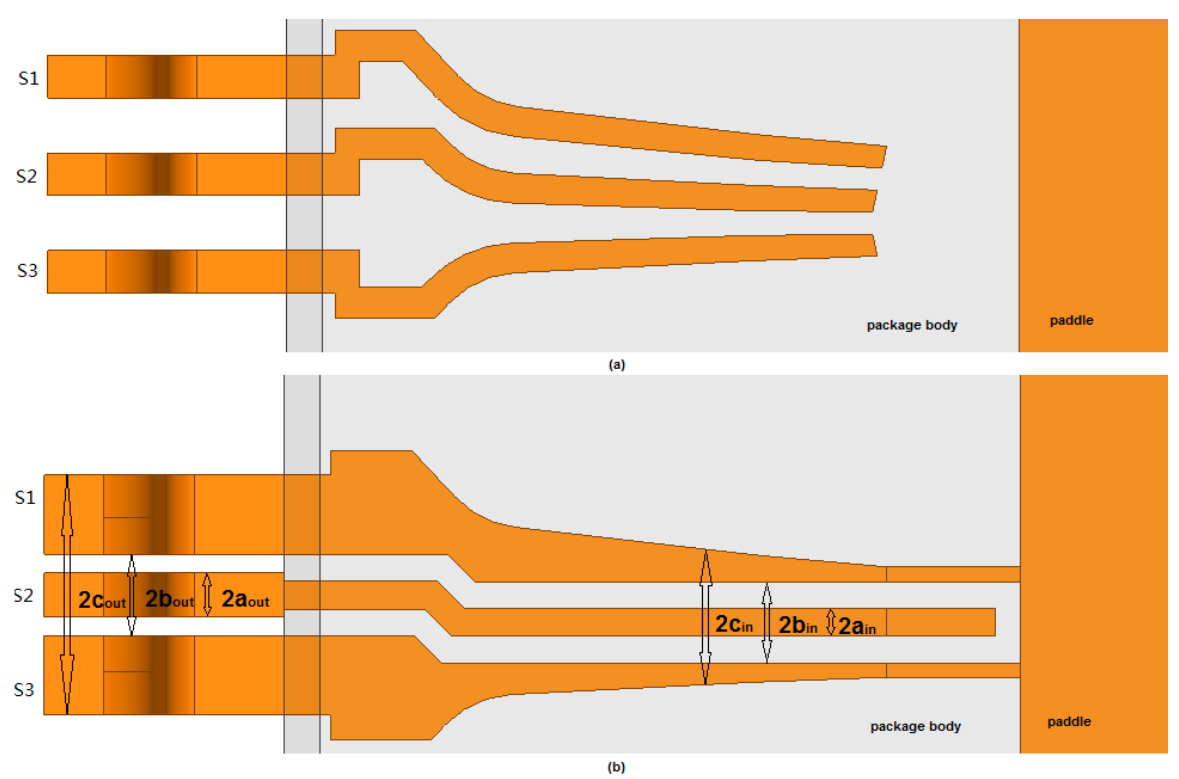

Figure 4. Two Cross-Sectional Views: (a) Traditional G-S-G Structure and (b) Proposed Coplanar Topology with Finite Irregular Ground Structure

In order to obtain the characteristic impedance of the coplanar transmission line structure in QFP80, we adopt quasi-static analysis to extract the transmission line capacitances, which can be used to derive the effective dielectric constant $\varepsilon_{\mathrm{e}}$ and characteristic impedance $\mathrm{Z}_{0}$. The classic expressions of $\mathrm{Z}_{0}$ and $\varepsilon_{\mathrm{e}}$ inside the package body are given as follows [5]:

$$
\begin{aligned}
\text { (1) } Z_{0} & =\frac{1}{v \sqrt{\varepsilon_{e}}\left(\mathrm{C}_{\mathrm{Ta}}+\mathrm{C}_{\mathrm{Ba}}+\mathrm{C}_{\mathrm{Pa}}\right)} \\
\text { (2) } \varepsilon_{\mathrm{e}} & =\frac{\mathrm{C}_{\mathrm{Ta}}+\mathrm{C}_{\mathrm{Td}}+\mathrm{C}_{\mathrm{Bd}}+\mathrm{C}_{\mathrm{Pd}}}{\mathrm{C}_{\mathrm{Ta}}+\mathrm{C}_{\mathrm{Ba}}+\mathrm{C}_{\mathrm{Pa}}}
\end{aligned}
$$

where:

$\mathrm{C}_{\mathrm{Ta}}$ - the per-unit-length capacitance produced by the top face of the leads without compound,

$\mathrm{C}_{\mathrm{Td}}$ - the per-unit-length capacitance produced by the top face of the leads with compound,

$\mathrm{C}_{\mathrm{Ba}}$ - the per-unit-length capacitance produced by the bottom face of the leads without compound,

$\mathrm{C}_{\mathrm{Bd}}$ - the per-unit-length capacitance produced by the bottom face of the leads with compound,

$\mathrm{C}_{\mathrm{Pa}}$ - the per-unit-length capacitance produced by the parallel plate of the leads without compound,

$\mathrm{C}_{\mathrm{Pd}}$ - the per-unit-length capacitance produced by the parallel plate of the leads with compound,

$\mathrm{V}$ - the speed of light in vacuum.

$\mathrm{C}_{\mathrm{Ta}}$ and $\mathrm{C}_{\mathrm{Td}}$ can be expressed as: 


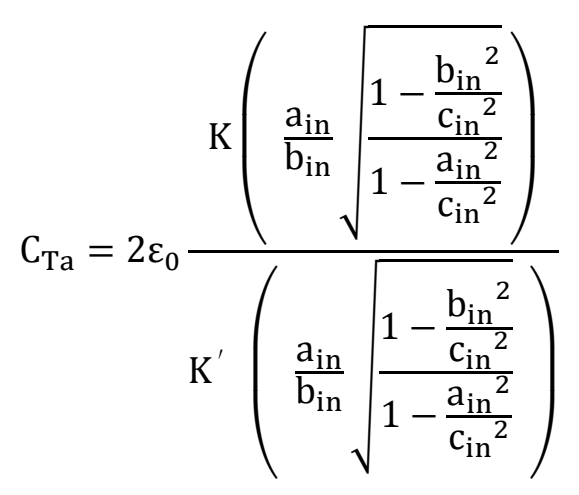

and



where:

$\mathrm{h}_{\mathrm{t}}, \mathrm{a}_{\mathrm{in}}, \mathrm{b}_{\mathrm{in}}, \mathrm{c}_{\mathrm{in}}$ - marked in figure3, figure 4 ,

$\varepsilon_{0}$ - the dielectric constant of vacuum,

$\varepsilon_{\mathrm{r}}$ - the dielectric constant of compound,

$\mathrm{K}, \mathrm{K}^{\prime}$ - the complete elliptic integrals and its complement.

The calculations of $\mathrm{C}_{\mathrm{Ba}}$ and $\mathrm{C}_{\mathrm{Bd}}$ are similar to the derivation of $\mathrm{C}_{\mathrm{Ta}}$ and $\mathrm{C}_{\mathrm{Td}}$. Furthermore, $\mathrm{C}_{\mathrm{Pa}}$ and $\mathrm{C}_{\mathrm{Pd}}$ can be calculated by:

$$
\mathrm{C}_{\mathrm{Pa}}=\frac{2 \varepsilon_{0} \mathrm{t}}{\mathrm{b}_{\mathrm{in}}-\mathrm{a}_{\text {in }}}
$$

and

$$
\mathrm{C}_{\mathrm{Pd}}=\mathrm{C}_{\mathrm{Pa}} \times \varepsilon_{\mathrm{r}}
$$

where: $\mathrm{t}$ - the thickness of lead.

The $\mathrm{Z}_{0}$ and $\varepsilon_{\mathrm{e}}$ outside the package body can be obtained similarly. 


\section{Construction of the Coplanar Transmission Line Structure}

In order to design the impedance of lead frame close to the characteristic impedance of the PCB transmission line, we adopt ANSYS HFSS to simulate and optimize the proposed coplanar transmission line structure [6,7]. According to Figure 4, we setup six configurations and simulate to find a better compromise between performance, robustness and fabrication, as shown in Table I. Figure 5 shows the simulation results of these configurations. From Figure 5 (a) and (b), the scenario \#2 shows better performance over the whole $20 \mathrm{GHz}$ bandwidth. Furthermore, from Figure 5 (c) and (d), the scenario \#6 with $0.130 \mathrm{~mm}$ spacing between the S1 and S2 (or S3 and S2) is best for impedance matching. But the $0.130 \mathrm{~mm}$ spacing is difficult to be fabricated. So, the physical configuration parameters of scenario \#2 and scenario \#5 can be combined to construct the cascade coplanar transmission line structure.

Table I. Simulation Configurations

\begin{tabular}{|c|c|c|c|c|}
\hline \multirow{2}{*}{ Configuration } & \multicolumn{2}{|c|}{$\begin{array}{c}\text { Inside part of } \\
\text { coplanar structure }\end{array}$} & \multicolumn{2}{c|}{$\begin{array}{c}\text { outside part of coplanar } \\
\text { structure }\end{array}$} \\
\cline { 2 - 5 } & $2 \mathrm{a}_{\text {in }}$ & $\mathrm{b}_{\text {in }}-\mathrm{a}_{\text {in }}$ & $2 \mathrm{a}_{\text {out }}$ & $\mathrm{b}_{\text {out }}-\mathrm{a}_{\text {out }}$ \\
\hline scenario \#1 & $0.20 \mathrm{~mm}$ & $0.230 \mathrm{~mm}$ & - & - \\
\hline scenario \#2 & $0.25 \mathrm{~mm}$ & $0.205 \mathrm{~mm}$ & - & - \\
\hline scenario \#3 & $0.30 \mathrm{~mm}$ & $0.180 \mathrm{~mm}$ & - & - \\
\hline scenario \#4 & - & - & $0.30 \mathrm{~mm}$ & $0.180 \mathrm{~mm}$ \\
\hline scenario \#5 & - & - & $0.35 \mathrm{~mm}$ & $0.155 \mathrm{~mm}$ \\
\hline scenario \#6 & - & - & $0.40 \mathrm{~mm}$ & $0.130 \mathrm{~mm}$ \\
\hline
\end{tabular}

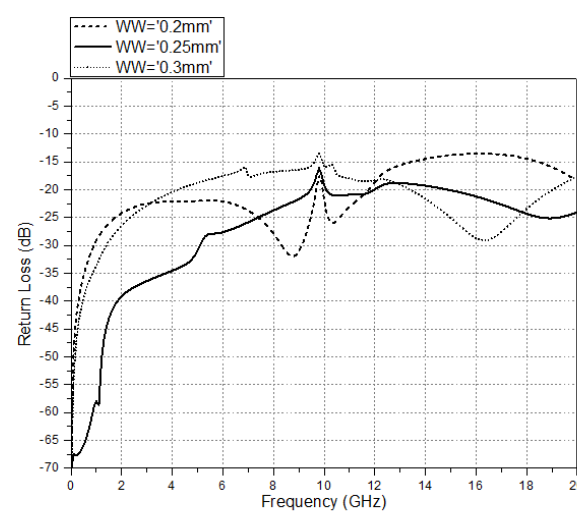

(a)

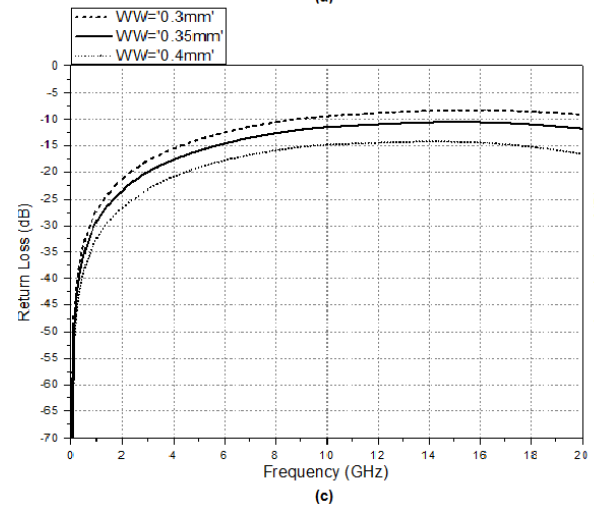

(c)

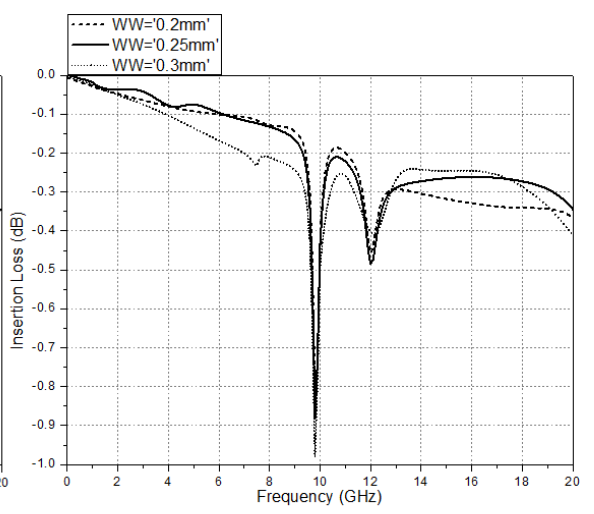

(b)

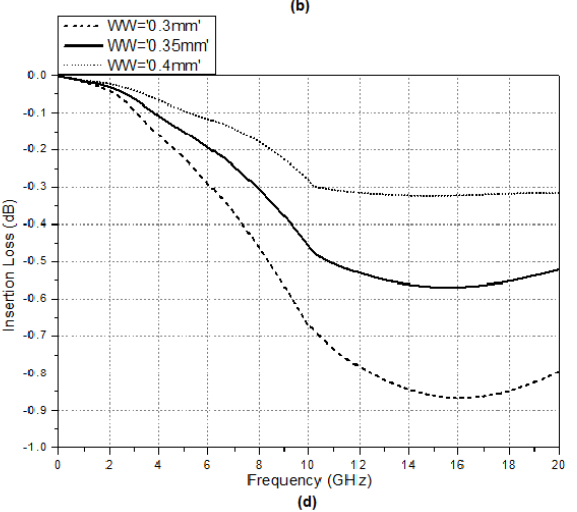

Figure 5. S-Parameter Sweep Results: (A) Return Loss of Inside Part, (B) Insertion Loss of Inside Part, (C) Return Loss of Outside Part, and (D) Insertion Loss of Outside Part 


\section{The Improved QFP80 Lead Frame Design based on the Coplanar Transmission Line Structure}

In this section, we use the optimal physical configuration parameters of the coplanar transmission line structure to complete the full improved QFP80 lead frame for high frequency $\mathrm{SiP}$ design based on figure1, and figure6 gives the corresponding schematic of this SiP. In Figure 6, there are four high-frequency channels (denoted by I, II, III and IV) in this SiP package. The channel I and II are designed for $5.8 \mathrm{GHz}$ signal transmission, and the channel III and IV are designed for $2.4 \mathrm{GHz}$ signal transmission. Figure7 shows the 2D design view of this improved QFP80 lead frame and the traditional QFP80 lead frame, respectively.

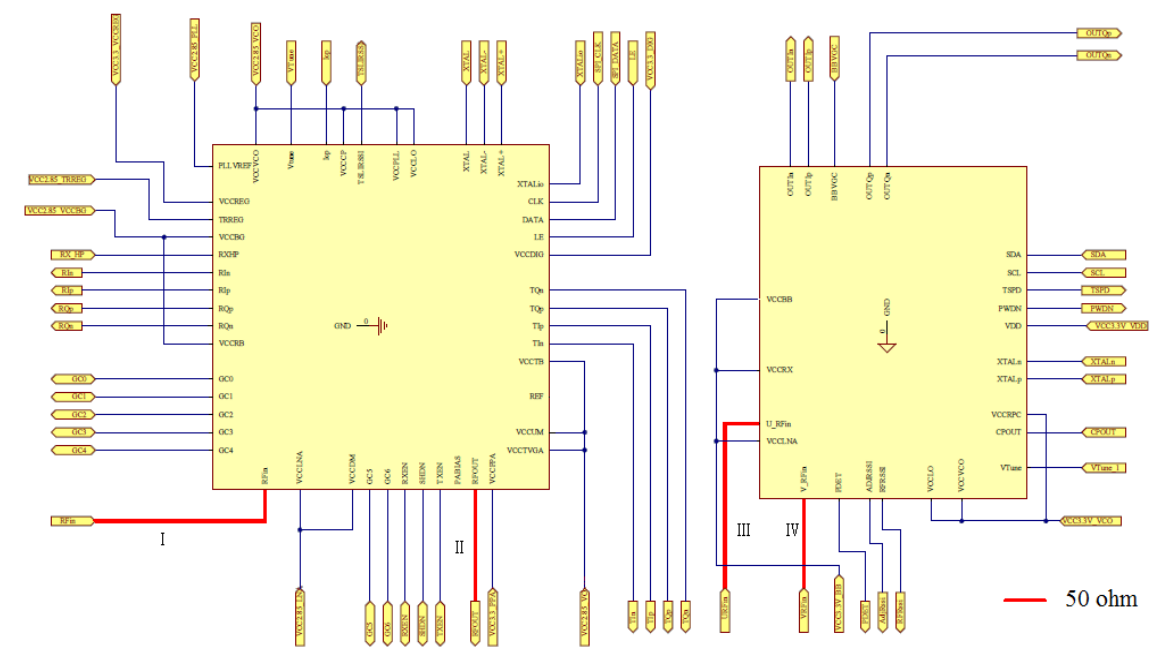

Figure 6. Schematic of SiP
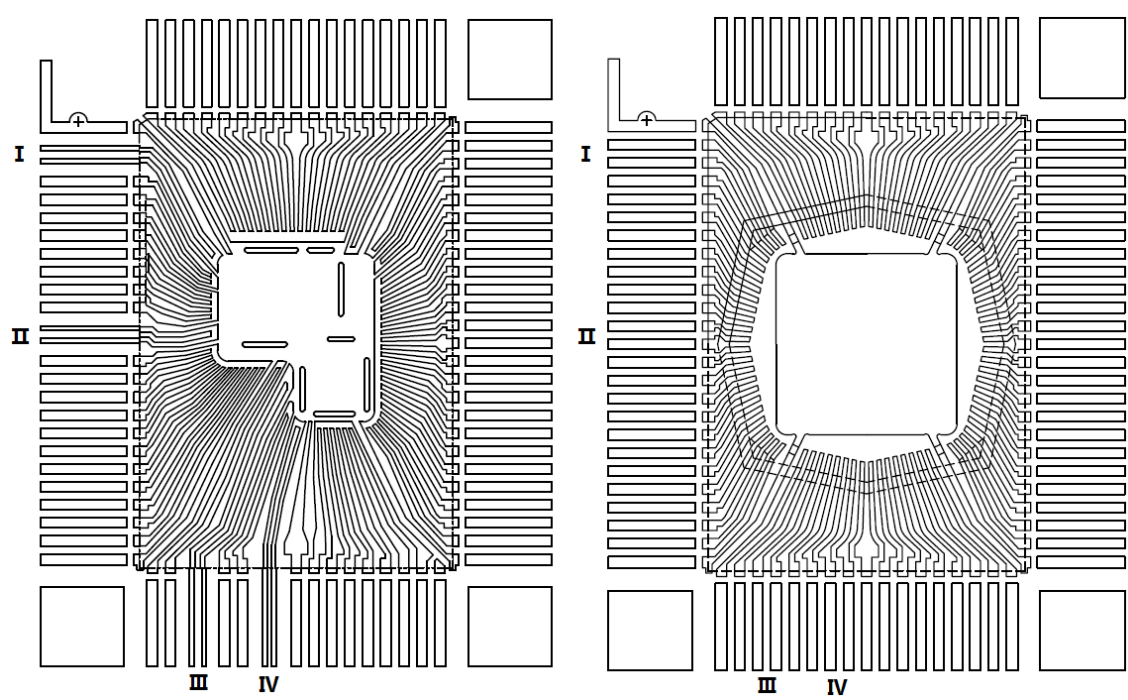

Figure 7. 2D Design View of this Improved QFP80 Lead Frame and Traditional QFP80 Lead Frame

In order to show the superiority of the improved QFP80 lead frame, we use HFSS to simulate these two structures. Table II gives the simulation configurations of the improved QFP80. It should be noted that the configuration of channel IV is different from others due to the compromise of fabrication. In order to evaluate the applicable frequency easily, we consider $-15 \mathrm{~dB}$ as criterion for bandwidth associated with return loss, and $-1 \mathrm{~dB}$ 
as criterion for insertion loss. Table III lists the summary of applicable frequency of these two structures. As we can see, all the channels in the improved structure show better impendence matching than corresponding channels in the traditional ground-signalground (G-S-G) structure, and the simulated results of channel I, II and III show good correlations between each other's. It should be noted that the performance of channel IV is degraded due to the different simulation configuration, but the bandwidth of it is adequate for $2.4 \mathrm{GHz}$ signal transmission. From above analysis, we can see that the cascade coplanar transmission line structure built into the QFP80 lead frame is remarkable for discontinuity cancellation.

Table II. Simulation Configurations of the Four Channels

\begin{tabular}{|c|c|c|c|c|}
\hline \multirow{2}{*}{ channel } & \multicolumn{2}{|c|}{$\begin{array}{c}\text { inside part of coplanar } \\
\text { structure }\end{array}$} & \multicolumn{2}{c|}{$\begin{array}{c}\text { outside part of coplanar } \\
\text { structure }\end{array}$} \\
\cline { 2 - 5 } & $2 \mathrm{a}_{\text {in }}$ & $\mathrm{b}_{\text {in }}-\mathrm{a}_{\text {in }}$ & $2 \mathrm{a}_{\text {out }}$ & $\mathrm{b}_{\text {out }}-\mathrm{a}_{\text {out }}$ \\
\hline $\mathrm{I}$ & $0.25 \mathrm{~mm}$ & $0.205 \mathrm{~mm}$ & $0.35 \mathrm{~mm}$ & $0.155 \mathrm{~mm}$ \\
\hline $\mathrm{II}$ & $0.25 \mathrm{~mm}$ & $0.205 \mathrm{~mm}$ & $0.35 \mathrm{~mm}$ & $0.155 \mathrm{~mm}$ \\
\hline $\mathrm{III}$ & $0.25 \mathrm{~mm}$ & $0.205 \mathrm{~mm}$ & $0.35 \mathrm{~mm}$ & $0.155 \mathrm{~mm}$ \\
\hline $\mathrm{IV}$ & $0.20 \mathrm{~mm}$ & $0.16 \mathrm{~mm}$ & $0.20 \mathrm{~mm}$ & $0.21 \mathrm{~mm}$ \\
\hline
\end{tabular}

Table III. Summary of Bandwidth for the Two Structures

\begin{tabular}{|c|c|c|c|c|}
\hline \multirow{2}{*}{ channel } & \multicolumn{2}{|c|}{ improved structure } & \multicolumn{2}{c|}{ traditional structure } \\
\cline { 2 - 5 } & $\mathrm{S} 11$ & $\mathrm{~S} 21$ & S11 & S21 \\
\hline I & $8.0 \mathrm{GHz}$ & $11.6 \mathrm{GHz}$ & $2.6 \mathrm{GHz}$ & $5.1 \mathrm{GHz}$ \\
\hline II & $9.4 \mathrm{GHz}$ & $15.2 \mathrm{GHz}$ & $2.8 \mathrm{GHz}$ & $6.1 \mathrm{GHz}$ \\
\hline III & $7.3 \mathrm{GHz}$ & $14.7 \mathrm{GHz}$ & $2.4 \mathrm{GHz}$ & $4.3 \mathrm{GHz}$ \\
\hline IV & $5.0 \mathrm{GHz}$ & $7.4 \mathrm{GHz}$ & $2.2 \mathrm{GHz}$ & $4.2 \mathrm{GHz}$ \\
\hline
\end{tabular}

\section{High Performance SiP Design based on the Improved QFP Lead Frame}

In this section, the high frequency SiP design based on the improved QFP80 lead frame is illustrated, as shown in Figure 8. Figure 8 (a) demonstrates the 3D design model of this improved SiP. In order to overview the characteristic impedance of the whole interconnect, we perform a TDR simulation of channel I. Figure 8 (b) shows the TDR simulation result of the channel I. From the TDR plot, characteristic impedance of each segment can be captured clearly. The impedance of improved lead segment has the maximum height of $63 \mathrm{ohm}$ (outside part) and the minimum height of 51ohm(inside part), respectively, and the impedance of the inductive wire bond has significantly reduced to $80 \mathrm{ohm}$. Furthermore, Figure 8 (c) and (d) show the simulated s-parameters against measured data of the selected channel I. Compared with Table III, the simulated bandwidth include improved lead and bond wire measured for S11 and S21 is decreased to $7.2 \mathrm{GHz}$ (at $-15 \mathrm{~dB}$ ) and $9.5 \mathrm{GHz}$ (at $-1 \mathrm{~dB}$ ). This is due to the influence of the inductive bond wires. From figure8 (c) and (d), we also can see that the return loss and insertion loss follow the same trends and show good agreements over the whole $20 \mathrm{GHz}$ bandwidth between the simulation and measurement. Due to the package processing error, the bandwidth measured for $\mathrm{S} 11$ and $\mathrm{S} 21$ is $6.3 \mathrm{GHz}$ (at $-15 \mathrm{~dB}$ ) and $8.3 \mathrm{GHz}$ (at $-1 \mathrm{~dB}$ ) from measured data, respectively. The results indicate that the improved QFP80 structure can be fully capable of supporting $5.8 \mathrm{GHz}$ high frequency $\mathrm{SiP}$ design, and the photograph of the improved SiP package is demonstrated in Figure 9. 


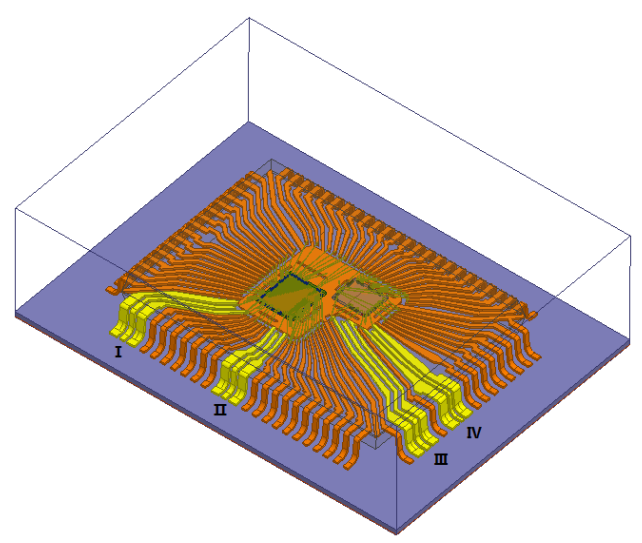

(a)

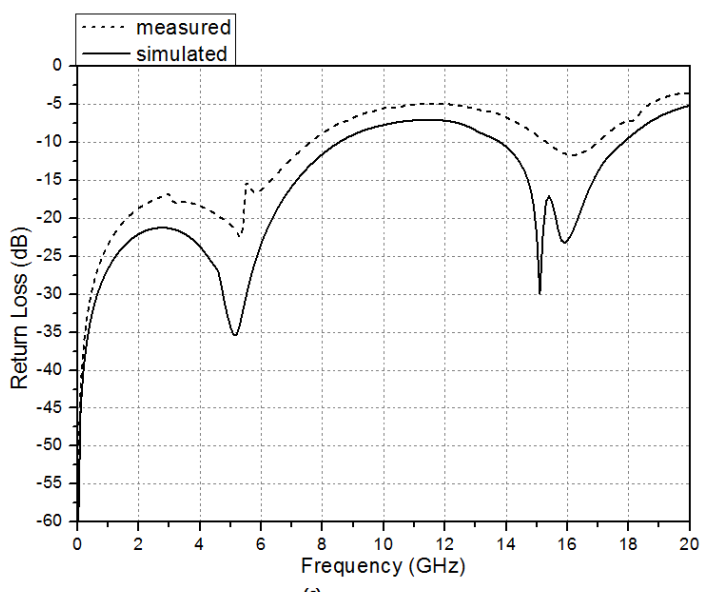

(c)

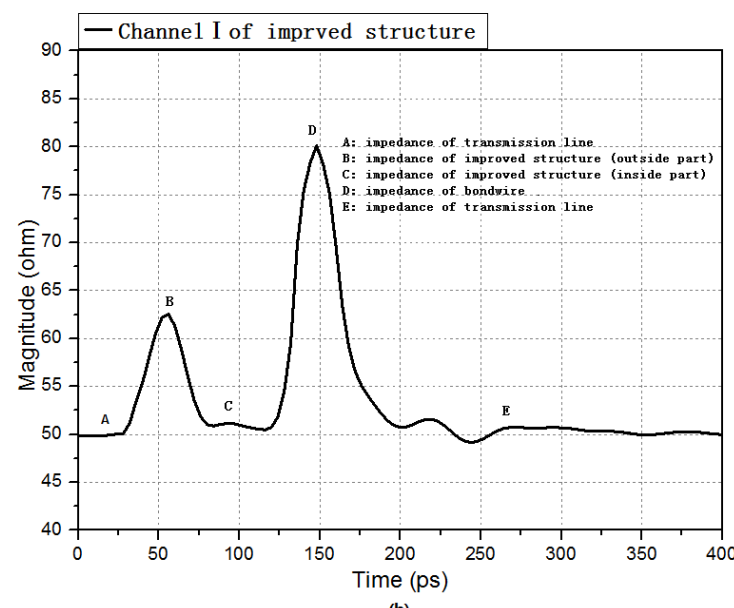

(b)

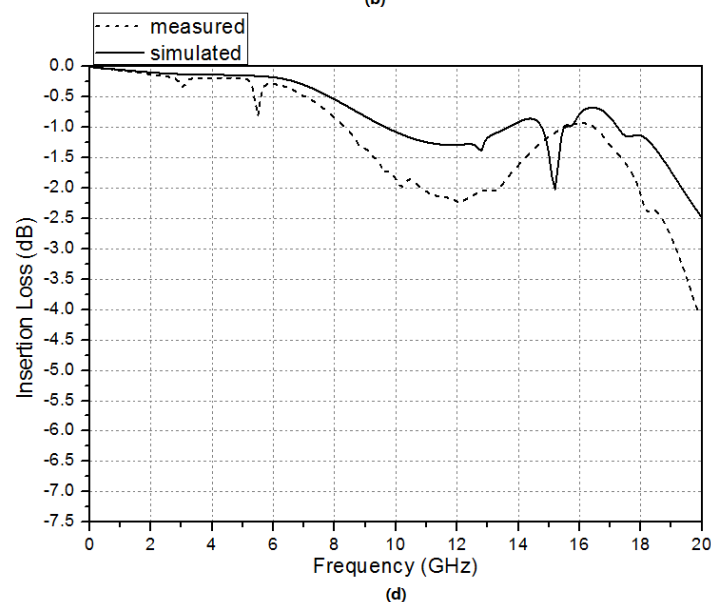

(d)

Figure 8. The High Frequency SiP Design based on the Improved QFP80 Lead Frame

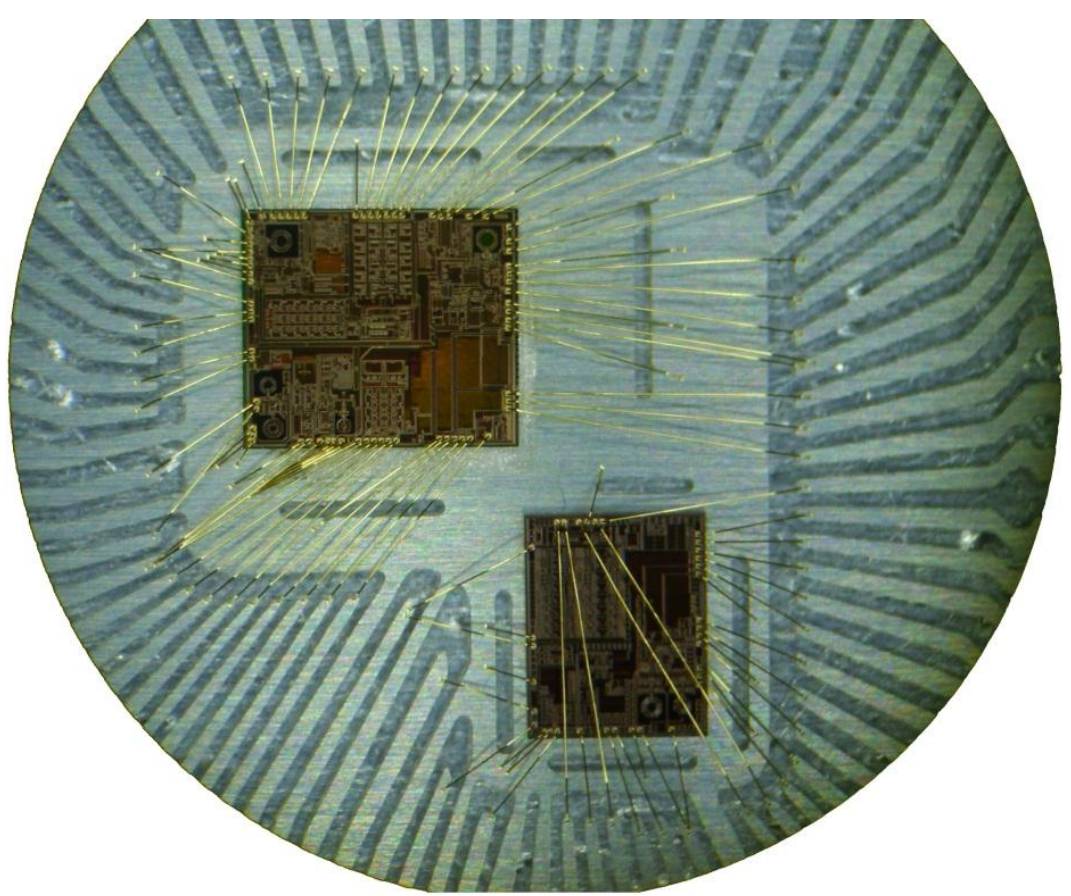

Figure 9. The Photograph of the New SiP 


\section{Conclusions}

In this paper, four cascade coplanar transmission line structures have been built in QFP80 lead frame for high frequency SiP application. The analysis results indicate that the improved QFP80 package is fully capable of supporting $5.8 \mathrm{GHz}$ high frequency SiP application. Because this improved structure needs layout space no more than traditional structure, it is suitable to be built in anywhere in QFP80 package. Additionally this design methodology could be readily applied not to only the QFP80 package but also to any other lead frame packaging technologies.

\section{Acknowledgments}

The research work is financially supported by the National Natural Science Foundation of China (No.61040032, No. 61306134) and the Educational Industrialization Foundation of Jiangsu Province (No. JHZD10-036).

\section{References}

[1] J.-H. Kim, R. Schmitt, D. Oh, W. Beyene, M. Li, A. Vaidyanath, J. Feng and C. Yuan, DesignCon 2009, Santa Clara, USA, (2009).

[2] Ryuichi and Oikawa, Proc. 60th Electronic Components and Technology Conference, Las Vegas, USA, (2010) June, pp. 1479-1484.

[3] J.-H. Kim, R. Schmitt, D. Oh, W. T. Beyene, M. Li, A. Vaidyanath, Y. Lu, J. Feng, C. Yuan, D. Secker and D. Mullen, Electronic Components and Technology Conference, (2009), pp. 1662-1669.

[4] T.-S. Horng, S.-M. Wu, H.-H. Huang, C.-T. Chiu and C.-P. Hung, IEEE Transactions on Microwave Theory and Techniques, vol. 49, no. 9, (2001), pp. 1538-1545.

[5] D. Jessie and L. Larson, Electronics Letters, vol. 37, no. 25, (2001) December, pp. 1521-1523.

[6] U. Paoletti, T. Hisakado and O. Wada, Proc. Electrical Design of Advanced Packaging and Systems Symposium, (2008) December, pp. 57-60.

[7] H. Sun, J. Wu and L. Sun, 12th International Conference on Electronic Packaging Technology \& High Density Packaging (ICEPT-HDP), (2011) August, pp. 573-576.

\section{Authors}

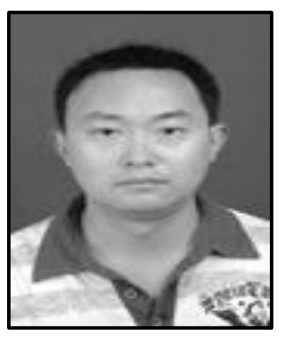

Haiyan Sun received the B.E. degree from Nantong University and the M.E. from Southeast University in 2000, and 2007, respectively. He has been a packaging engineer since 2004. He is responsible for advanced IC packaging development, IC package design and substrate layout, and thermal and signal integrity analysis of IC packages. Since 2009, he has been a Doctoral Candidate at the department of National ASIC System Engineering Research Center, Southeast University, Nanjing, China, working on low-cost system in package research.

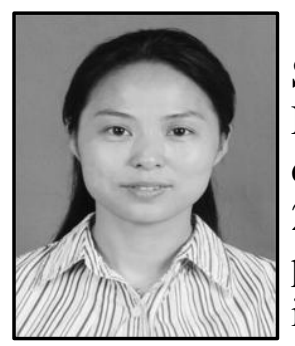

Ling Sun was born in Jiangsu Province, China, in 1976. She received the B.E. degree from Nantong University, Nantong, in 1998. She earned the M.E. and the Ph.D. degrees from Southeast University, Nanjing, China, in 2004 and 2007. She is currently working as an associate professor at Nantong University. Her research interests include device modeling, RFIC design, packaging design and modeling, and EMC issues. 